JOURNAL OF SYNCHROTRON RADIATION

ISSN 1600-5775

Received 13 September 2016

Accepted 24 October 2016

Edited by S. M. Heald, Argonne National Laboratory, USA

Keywords: inelastic X-ray scattering; direct tomography; diamond anvil cell; high pressure.

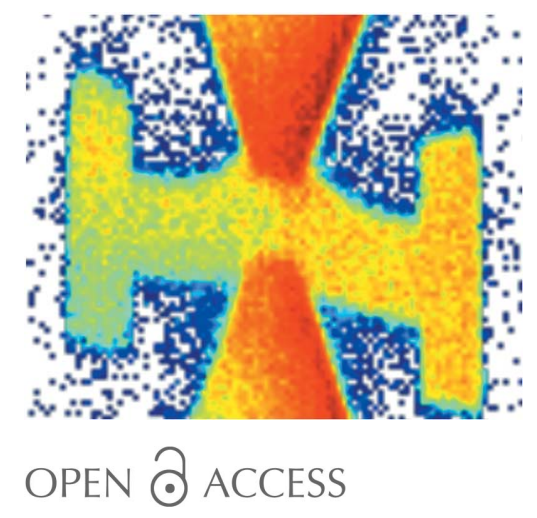

\section{Direct tomography imaging for inelastic X-ray scattering experiments at high pressure}

\author{
Ch. J. Sahle, ${ }^{a *}$ A. D. Rosa, ${ }^{a}$ M. Rossi, ${ }^{a}$ V. Cerantola, ${ }^{a}$ G. Spiekermann, \\ S. Petitgirard, ${ }^{\text {J J. Jacobs, }}{ }^{\text {S }}$ S. Huotari, ${ }^{\text {d }}$ M. Moretti Sala ${ }^{a}$ and A. Mirone ${ }^{a}$
}

\begin{abstract}
${ }^{\mathbf{a}}$ European Synchrotron Radiation Facility, 71 Avenue des Martyrs, 38000 Grenoble, France, ${ }^{\mathbf{b}}$ Institute of Earth and Environmental Science, Universität Potsdam, Potsdam, Germany, ${ }^{\mathbf{c} B a y e r i s c h e s ~ G e o i n s t i t u t, ~ U n i v e r s i t y ~ o f ~ B a y r e u t h, ~}$ Bayreuth, Germany, and ${ }^{\mathbf{d}}$ Department of Physics, POB 64, FI-00014, University of Helsinki, Helsinki, Finland. *Correspondence e-mail: christoph.sahle@esrf.fr
\end{abstract}

A method to separate the non-resonant inelastic X-ray scattering signal of a micro-metric sample contained inside a diamond anvil cell (DAC) from the signal originating from the high-pressure sample environment is described. Especially for high-pressure experiments, the parasitic signal originating from the diamond anvils, the gasket and/or the pressure medium can easily obscure the sample signal or even render the experiment impossible. Another severe complication for high-pressure non-resonant inelastic X-ray measurements, such as X-ray Raman scattering spectroscopy, can be the proximity of the desired sample edge energy to an absorption edge energy of elements constituting the DAC. It is shown that recording the scattered signal in a spatially resolved manner allows these problems to be overcome by separating the sample signal from the spurious scattering of the DAC without constraints on the solid angle of detection. Furthermore, simple machine learning algorithms facilitate finding the corresponding detector pixels that record the sample signal. The outlined experimental technique and data analysis approach are demonstrated by presenting spectra of the $\mathrm{Si} L_{2,3}$-edge and $\mathrm{O} K$-edge of compressed $\alpha$-quartz. The spectra are of unprecedented quality and both the $\mathrm{O} K$-edge and the $\mathrm{Si}$ $L_{2,3}$-edge clearly show the existence of a pressure-induced phase transition between 10 and $24 \mathrm{GPa}$.

\section{Introduction}

Non-resonant inelastic X-ray scattering from core electrons, or X-ray Raman scattering (XRS) spectroscopy, has proven a valuable tool for the study of shallow electronic absorption edges under extreme pressure and temperature conditions (Mao et al., 2001; Rueff \& Shukla, 2010; Sternemann \& Wilke, 2016). For example, it has recently been used to study highpressure phases of water and ice (Mao et al., 2006; Tse et al., 2008; Pylkkänen et al., 2010; Sahle et al., 2013), pressureinduced transitions in electron topology (Tse et al., 2011, 2014) and pressure-induced changes in the electronic structure of condensed matter in general (Shieh et al., 2013). Even valence electron excitations are accessible (Loa et al., 2011).

XRS possesses the unique strength to probe shallow electronic absorption edges of light elements such as oxygen and silicon, using hard X-rays that easily penetrate several millimeters of diamond (Sternemann et al., 2013). In this respect, XRS is the sibling of soft X-ray absorption spectroscopy (Henderson et al., 2014) and electron energy-loss spectroscopy, suited to investigate samples contained inside diamond anvil cells. Thus, the direct investigation of the local structure of even amorphous materials at high pressure, or of the 
amorphization of initially crystalline material, is at hand. However, there are several drawbacks to overcome. The scattering cross section is orders of magnitude lower compared with that of resonant techniques, such as X-ray absorption, X-ray emission and resonant inelastic X-ray scattering spectroscopy. In addition, the overall measured XRS signal contains the scattering contributions of all electrons located along the X-ray beam pathway. For high-pressure experiments, the parasitic signal originating from the sample environment (the diamond anvils, gasket material or pressure medium) can, therefore, obscure the sample signal or even render the experiments impossible, especially so if the sample environment exhibits core-level excitations in the vicinity of the desired edge of the sample; for example, the carbon $K$-edge from diamond or the Be $K$-edge from the frequently used Be high-pressure gaskets. In order to cope with these difficulties, it is important to utilize brilliant X-ray sources, an efficient detection scheme (large solid angle of detection) and an efficient procedure for the separation of the desired signal from the spurious scattering originating from the high-pressure sample environment. In recent years, developments and improvements of XRS have blossomed at synchrotron facilities, which indicates an increased interest and potential for this technique. Implementation of bentcrystal analyzers have considerably improved the energy resolution (Hiraoka et al., 2013). The separation of the scattering signal from the sample and its container have been improved with the use of focusing polycapillary optics with, however, a limitation of the solid angle collection of the XRS spectrometer (Chow et al., 2015). Finally, improvements in the high-pressure technique dedicated to XRS have extended the accessible pressure range with improved data quality (Hiraoka et al., 2016; Petitgirard et al., 2017).

Here, we introduce an experimental setup and a data analysis method that aids separating the desired sample signal and the scattering background arising from the sample container, such as in a DAC, without constraining the detectable solid angle. We demonstrate the potential of this approach by presenting oxygen $K$ - and silicon $L_{2,3}$-edge data of an unprecedented quality of the pressure-induced amorphization and coordination change of $\alpha$-quartz. After a short summary of the XRS theory and background in $\$ 2$, we will describe in detail our experimental setup and data analysis scheme in $\$ 3$. Data from the $\mathrm{O} K$ - and $\mathrm{Si} L_{2,3}$-edge of $\mathrm{SiO}_{2}$ quartz at high pressure obtained with this setup are presented in section $\S 4$, and, finally, $\S 5$ gives a summary and outlook for our new procedure.

\section{Theoretical background}

$\mathrm{X}$-ray Raman scattering is non-resonant inelastic X-ray scattering from core electrons. The measured quantity in an XRS experiment (like in all non-resonant inelastic X-ray scattering experiments) is the double differential scattering cross section (Schülke, 2007)

$$
\frac{\mathrm{d}^{2} \sigma}{\mathrm{d} \Omega \mathrm{d} \omega}=\left(\frac{\mathrm{d} \sigma}{\mathrm{d} \Omega}\right)_{\mathrm{Th}} S(\mathbf{q}, \omega) .
$$

Here, $(\mathrm{d} \sigma / \mathrm{d} \Omega)_{\mathrm{Th}}$ is the Thomson scattering cross section

$$
\left(\frac{\mathrm{d} \sigma}{\mathrm{d} \Omega}\right)_{\mathrm{Th}}=r_{\mathrm{e}}^{2} \frac{\omega_{2}}{\omega_{1}}\left(\varepsilon_{1} \cdot \varepsilon_{2}\right),
$$

with the classical electron radius $r_{\mathrm{e}}$ and the polarization vectors of the incident $\left(\varepsilon_{1}\right)$ and scattered beam $\left(\varepsilon_{2}\right)$, and $S(\mathbf{q}, \omega)$ is the so-called dynamic structure factor

$$
S(\mathbf{q}, \omega)=\sum_{\mathrm{i}, \mathrm{f}} p_{\mathrm{i}}|\langle\mathbf{f}|\exp (i \mathbf{q} \cdot \mathbf{r})| \mathrm{i}\rangle|^{2} \delta\left(E_{\mathrm{i}}-E_{\mathrm{f}}+\omega\right) .
$$

In the above equations, $\omega=\omega_{1}-\omega_{2}$ is the energy transferred to the sample system during the inelastic scattering event, where $\omega_{1}$ is the energy of the incident photon beam and $\omega_{2}$ is that of the scattered photons. The sum in (3) is over all final states $|\mathbf{f}\rangle$ and all initial states $|\mathrm{i}\rangle$ weighted by their corresponding probability $p_{\mathrm{i}}$. Note that besides the energy transfer $\omega$, a momentum $\mathbf{q}$ is transferred to the sample system which, because XRS utilizes hard X-rays, can be considerable. $S(\mathbf{q}, \omega)$ holds all information about the scattering sample obtainable by XRS. As only the transferred energy $\omega$ is important in XRS, the incident energy can be chosen freely, which makes it especially suitable to study absorption edges of low- $Z$ element containing materials under in situ conditions. Commonly used incident energies are of the order of $10 \mathrm{keV}$, whereas the energy transfer is of the order of low- $Z$ absorption edges (e.g. the carbon or oxygen $K$ - or silicon $L$-edges). It was shown by Mizuno and Ohmura that, at low transferred momenta, $S(\mathbf{q}, \omega)$ is directly proportional to the photoelectric absorption spectra typically measured with soft X-ray absorption spectroscopy (Mizuno \& Ohmura, 1967). A detailed overview of XRS and other inelastic X-ray scattering techniques is given by Schülke (2007).

Despite the fact that the utilized hard X-rays can penetrate even very complex sample environments, such as the DACs used for generating high pressures, XRS from samples contained inside DACs has several drawbacks. Because XRS is non-resonant scattering of X-rays, the measured signal contains the inelastic response of all electrons in the interaction pathway of the incoming X-ray beam: the sample and the sample environment. The spurious signal from the DAC can arise from the valence electrons of the sample environment, either collective valence excitations (dominating at low energy and momentum transfers) or Compton scattering (dominating at larger energy and momentum transfers) (Sahle et al., 2015). Often, the signals originating from these valence electron excitations vary smoothly as a function of energy loss over the range of interest and, if kept small enough, can be subtracted from the measured signal using parameterized functions (Sternemann et al., 2008). However, typical sample sizes in high-pressure experiments are a few tens of micrometers compared with millimeters of the diamond anvils or the high-pressure gasket material (e.g. Be, Rh, BN). In more unfortunate cases, the shallow absorption edge of interest coincides with an absorption edge of the DAC. This, for 
example, is the case when measuring the $\mathrm{Si} L_{2,3}$-edge (edge onset at approximately $100 \mathrm{eV}$ in elemental $\mathrm{Si}$ and approximately $105 \mathrm{eV}$ in $\mathrm{SiO}_{2}$ ) through a Be gasket ( $K$-edge onset of Be at $111.5 \mathrm{eV}$ ).

\section{Experimental methods}

\subsection{High-pressure technique and sample description}

To generate high-pressure conditions between ambient and $24 \mathrm{GPa}$, we used a newly designed panoramic DAC (Fig. 1a). The panoramic DAC was equipped with adapted extra-high conical diamond anvils (Boehler \& De Hantsetters, 2004) (diameter of $3.1 \mathrm{~mm}$ and height of $2.72 \mathrm{~mm}$ ) with a culet size of $500 \mu \mathrm{m}$. The opening angles of the cell were $80^{\circ}$ from the compression axis and $140^{\circ}$ in the plane perpendicular to the compression axis.

The new panoramic DAC and its adapted extra-high conical diamond anvils have been designed by the ESRF, Sample Environment Support Service-HP, and the cell is available for all ESRF users via this service. For the gasket material, we used a custom-machined Be gasket (5 mm outer diameter), pre-indented to approximately $50 \mu \mathrm{m}$ thickness, and laserdrilled a hole of $250 \mu \mathrm{m}$ diameter. This hole was then filled with amorphous B-epoxy (a-BE) (a-B:epoxy-resin:hardener ratio was $1: 0.22: 0.08$ by weight), compressed and drilled again for a final sample chamber of $150 \mu \mathrm{m}$ diameter (Merkel \& Yagi, 2005; Sato et al., 2013; Rosa et al., 2016). The resulting a-BE ring around the sample serves two purposes: firstly, it stabilizes the hole in the Be gasket and, secondly, it aides in the spatial separation between the sample and the Be gasket. The latter is important because the spatial imaging resolution is currently limited to approximately $50-100 \mu \mathrm{m}$ by the analyzer focus and the detector pixel size.

The sample chamber was filled with pure $\alpha-\mathrm{SiO}_{2}$ quartz together with two ruby spheres that we positioned at the side of the sample chamber to avoid recording the $\mathrm{O} K$-edge of the ruby spheres. Pressure calibration was obtained before and after each measurement using the standard ruby fluorescence method (Mao et al., 1986).
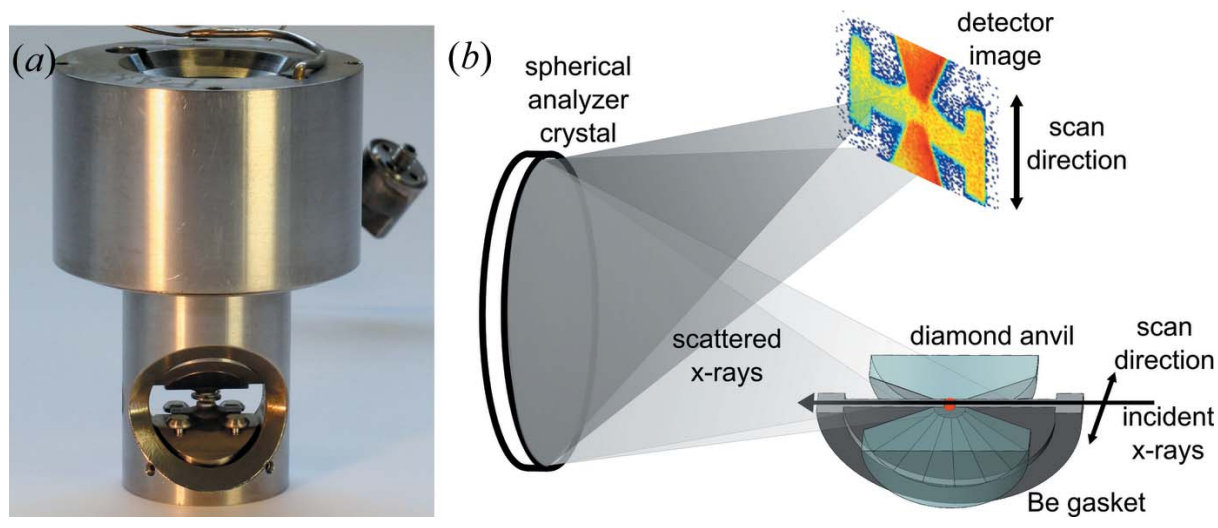

Figure 1

(a) Photograph of the membrane-driven panoramic diamond anvil cell. (b) Schematic demonstrating the imaging properties: X-rays scattered at different positions along the beam are focused in a one-toone fashion by the spherically bent $\mathrm{Si}$ analyzer crystal onto different positions on the detector.

\subsection{Beamline setup}

All XRS data were recorded at the inelastic scattering beamline ID20 of the ESRF, Grenoble, France. The incident beam was monochromated using an $\mathrm{Si}(111)$ high-heat-load monochromator and a succeeding $\mathrm{Si}(311)$ channel-cut postmonochromator. With a mirror system in Kirkpatrick-Baez geometry, we focused the beam to a spot size of $10 \times 20 \mu \mathrm{m}$ at the sample position. The inelastically scattered radiation was analyzed using 12 spherically bent $\mathrm{Si}(660)$ crystals and detected using a Maxipix detector (pixel size $55 \mu \mathrm{m}$ ) (Ponchut et al., 2011). The overall energy resolution was $0.7 \mathrm{eV}$ as estimated from the full width at half-maximum of the quasielastic line of a piece of Scotch tape prior to the highpressure experiment.

\subsection{XRS-based imaging at high pressures}

To separate the desired weak scattering signal from the sample and the strong spurious scattering originating from the DAC, we use the inherent imaging capabilities of a setup with bent $\mathrm{Si}$ analyzer crystals and pixelated area detectors (Huotari et al., 2011). The bent analyzer crystals provide a point-topoint focus of the photon beam as it penetrates through the sample environment and the sample. This is illustrated schematically in Fig. 1(b): within the field of view of each analyzer crystal, each point along the incident X-ray beam is focused onto a different point on the detector. A detailed description of this imaging technique was given by Huotari et al. (2011). A setup following this scheme is currently installed at ID20 of the ESRF. The contrast for the generated image is given by the dynamic structure factor $S(\mathbf{q}, \omega)$ and, thus, can be chosen freely from the spectrum, for example, the electron density $[S(\mathbf{q}, 0)]$, valence electronic excitations $\left[S\left(\mathbf{q}, w_{\mathrm{p}}\right)\right.$, where $w_{\mathrm{p}}$ is the plasmon frequency], or the core edges $\left[S\left(\mathbf{q}, w_{\mathrm{e}}\right)\right.$, where $w_{\mathrm{e}}$ is an energy loss above the respective core edge onset].

These imaging capabilities can be exploited in two ways. Firstly, scanning the sample through the incident X-ray beam at a fixed energy, one can create a two-dimensional image corresponding to a cut through the sample in the scanned direction. The spatial resolution of these images is given by the size of the incident X-ray beam in the two directions perpendicular to the incident beam, the pixel size of the area detector and the focus/response of the analyzer crystal. Two examples of these two-dimensional images are shown in Figs. 1(a) and 1(b). Fig. 1(a) shows the image of a horizontal sample scan, where one can clearly see the high contrast from the diamond anvils compared with the Be gasket material. Fig. 1(b) shows a vertical alignment scan, which in the used geometry corresponds to a cut through the Be gasket and one can clearly see the sample as a small red circle in the 


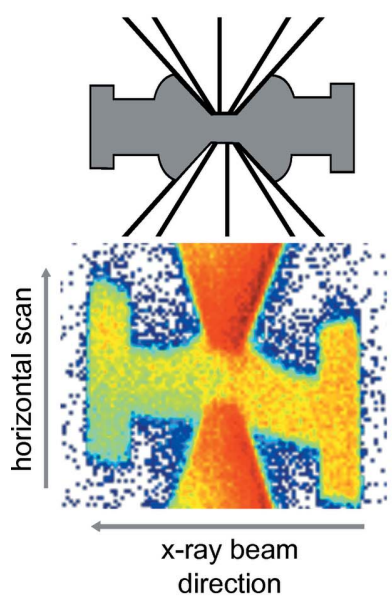

(a)

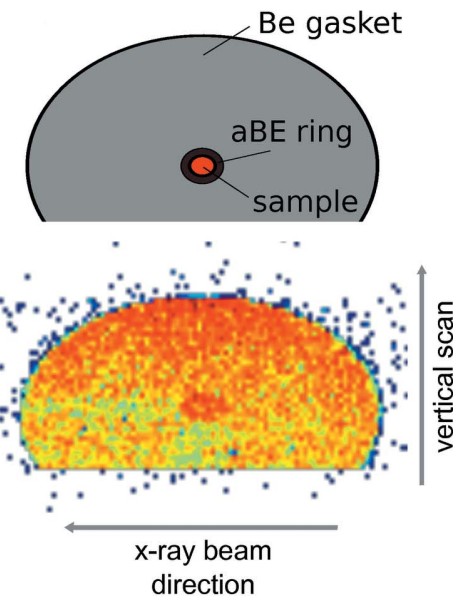

(b)
Figure 2

Schematics (top row) and actual constructed two-dimensional images of alignment scans in the horizontal direction $(a)$ and vertical direction $(b)$, respectively. The X-ray energy was set to the top of the elastic line at $9.7 \mathrm{keV}$ [i.e. the contrast is given by $S(q, 0)]$

center of the gasket. These two-dimensional images are, thus, extremely useful when aligning the sample as the scale along the scanning direction (denoted 'horizontal scan' and 'vertical scan' in Fig. 2) is given by the absolute motor positions of the instrument.

Secondly, once the sample is well aligned in the incident beam, we can exploit the imaging properties of the spectrometer during the actual XRS measurements. The spatial information along the beam can be used to select pixels on the detector that only record scattered intensity from the sample, whereas intensity from pixels that record spurious scattering originating from the DAC (gasket, diamond or pressure medium) can be discarded. The great advantage of this approach is that it can be applied during the detailed data analysis after the experiment has been performed. It imposes no restrictions on the amount of solid angle covered by the spectrometer and it renders the alignment of a complicated post-sample optic unnecessary.

Naturally, the question arises of how to select detector pixels associated with scattering from the sample as opposed to the sample environment. In many cases, especially for relatively large samples (e.g. powder samples) and for samples with a large density contrast compared with the high-pressure cell, the selection of pixels is straightforward and can be accomplished manually. Often, varying the contrast by choosing different energy-loss values (e.g. below and above an absorption edge) can significantly facilitate the selection of appropriate regions of interest (ROIs).

Nevertheless, certain cases pose significant experimental challenges. The density contrast between the sample and the gasket material may be very low or, even more severe, if pixels that record scattering from the environment have to be avoided at all cost, e.g. if the DAC material exhibits an absorption edge in the energy-loss region of interest. As already mentioned above, this is the case for the $K$-edge of carbon, where scattering from the diamond anvils can obscure completely the weak signal of a carbon-containing sample, or the $L_{2,3}$-edge of silicon, which coincides with the $K$-edge of the often used gasket material Be.

\subsection{ROI selection}

The scattering signals were analyzed using the XRStools software package (Sahle et al., 2015). For the identification of those detector pixels that record scattering from the sample as opposed to scattering from the Be gasket, we used a matrix factorization scheme that we newly implemented in the XRStools package: we defined rough ROIs manually, constructed a spectrum for each of the pixels inside these rough ROIs, used a factorization scheme [such as non-negative matrix factorization (NNMF), independent component analysis or principal component analysis] and choose the component spectrum that corresponds to the desired sample spectrum. In a last step, the algorithm calculates the covariance of the spectra from each of the pixels inside the rough ROI and the chosen component and discards pixels that have recorded spectra with a too low covariance. We note that instead of calculating the covariance between the chosen spectrum and each spectrum inside the rough ROI, any other measure of similarity may also be applicable. The described scheme can be iterated several times until the ROI consists only of pixels that record solely the scattering from the sample. For the matrix factorization, we utilize the schemes available in the Scikit-learn Python module (Pedregosa et al., 2011). This ROI-finding approach is illustrated in Fig. 3, and Fig. 3(a) shows the result of the described matrix factorization for a single ROI using NNMF. Here, we used the signal from the $\mathrm{O}$ $K$-edge; however, the signal from the $\mathrm{Si} L_{2,3}$-edge or, if several iterations are necessary, a combination of both edges is also suitable. The second component (solid red line) already starkly resembles the oxygen $K$-edge, whereas the first component (blue line) only shows a linear background. Even though the named factorization schemes are mathematically well defined, they are not guaranteed to yield physically meaningful results. However, for the purpose of finding the correct pixels inside a given rough ROI, this approach is well suited and, as was shown earlier (Inkinen et al., 2015; Sahle et al., 2016; Niskanen et al., 2016), under certain circumstances, NNMF components can directly be related to physical spectra. In Fig. 3(b), we plot the covariance between the spectrum of each pixel of the ROI with the desired component 2 of the matrix factorization. The horizontal line represents the threshold we chose to discard pixels exhibiting low-covariance spectra. Fig. 3(c) shows the Si $L_{2,3}$-edge spectra after utilizing the ROIs from the different stages of the data extraction. A series of image representations of the ROI at the different iterations is shown as an inset in Fig. 3(c): the top picture (ROI iteration 0 ) represents the ROI as chosen manually. Here, the red region corresponds to pixels of the chosen ROI, the next two pictures show the refined ROI after iterations one and two using NNMF and consecutive thresholding, respectively. In the final step, only a single pixel is left as an ROI. 


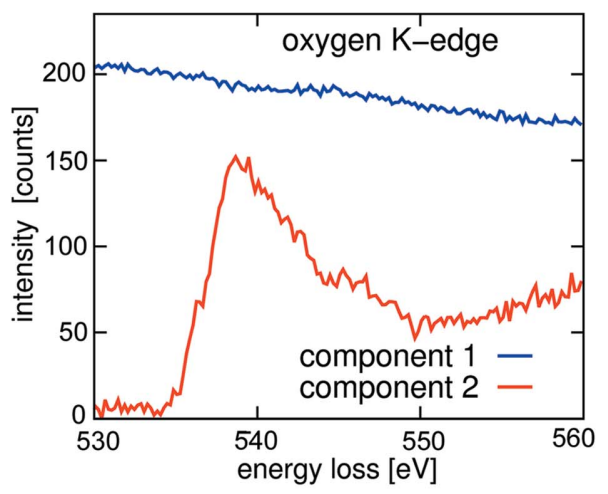

(a)

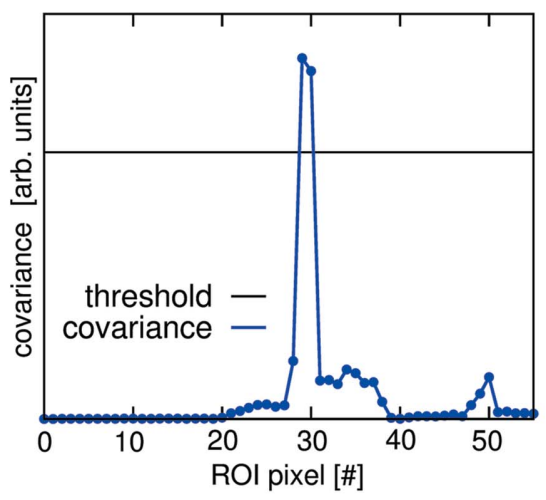

(b)

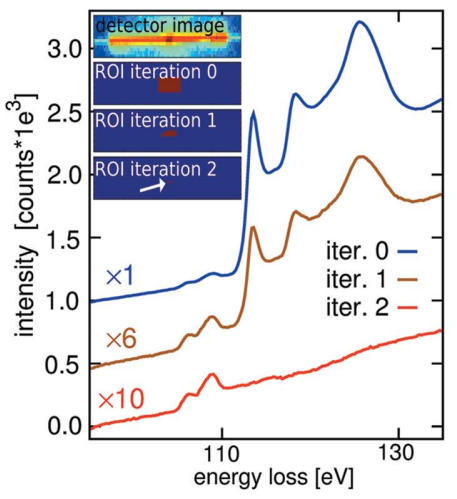

(c)

Figure 3

(a) The two main components of a non-negative matrix factorization from a single ROI computed from a scan of the $\mathrm{O} K$-edge. Component 1 is the major component and corresponds to the many pixels that record scattering from the Be gasket. Component 2 corresponds to pixels that record scattering from the $\mathrm{SiO}_{2}$ sample. (b) Covariance of the spectrum of each respective pixel and the spectrum of component 2 from part $(a)$. In a first iteration, we chose a threshold represented by the horizontal black line. (c) Image representation of the ROI used to initiate the NNMF (inset top), the resulting ROI after the thresholding shown in $(b)$, the single pixel ROI after two iterations of the NNMF procedure, and the corresponding detector image (inset bottom) used in the NNMF demonstration in part $(b)$ and $(c)$.

\section{Pressure-induced changes in the local atomic environment of $\alpha$-SiO}

\subsection{Background}

One of the great potentials of high-pressure XRS is the ability to probe the local electronic environment of low- $Z$ elements even in disordered material, providing essential data on the structure and properties of amorphous compounds under high-pressure conditions (Sternemann et al., 2013). Phenomena such as the pressure-induced amorphization in silicate minerals (Daniel et al., 1997; Hemley et al., 1988) or back transformation of high-pressure polymorphs to glassy silicates as found in lunar meteorites (Ohtani et al., 2011) are of extreme importance to understand the time-scale and peak pressure of shock conditions in the early solar system (Beck et al., 2005; Kubo et al., 2010). Similarly, the transformations and behavior of silicate glasses at high pressure raised considerable attention because they can be used to mimic and interpret the fate of melts in the deep Earth (Meade \& Jeanloz, 1988; Sato \& Funamori, 2008; Sanloup et al., 2013; Murakami \& Bass, 2011; Petitgirard et al., 2015). However, a comprehensive understanding between the bulk properties of amorphous matter and the underlying atomic scale structure under high pressure is still missing.

For instance, the coordination change upon compression in $\mathrm{SiO}_{2}$ glass, with an increase of the number of oxygen atoms from four to six in the first coordination shell around silicon, is still a matter of debate (Murakami \& Bass, 2010; Sato \& Funamori, 2010; Wu et al., 2012). Also, the pressure-induced amorphization of quartz is poorly constrained due to a lack of experimental data on such high-pressure phase transformations. So far, these questions have been addressed mainly using X-ray diffraction (Sanloup et al., 2013; Sato \& Funamori, 2010; Benmore et al., 2010) or optical probes such as Raman (Hemley et al., 1986; Shim \& Catalli, 2009) or Brillouin (Murakami \& Bass, 2011; Sanchez-Valle \& Bass, 2010) spectroscopy. However, these techniques give information on the intermediate range structure and are not element-specific. Thus, data on the local electronic environment and structure of the main constituent of silicates ( $\mathrm{Si}, \mathrm{O}, \mathrm{Mg}, \mathrm{Al}$, etc.) can shed new light on the behavior of glasses and the induced amorphization of silicates at high pressure. The local electronic structure, reflecting the local coordination, has remained inaccessible at high pressure because, for example, the signal of soft X-ray absorption spectroscopy or electron energy-loss spectroscopy is fully attenuated in the first micrometers of the anvils or gaskets in DACs.

\subsection{O K-edge}

The spectra extracted using the refined ROIs are presented in Fig. 4. Fig. 4(a) shows the $\mathrm{O} K$-edge data measured at 0,10 and $24 \mathrm{GPa}$, and a spectrum of the $\mathrm{O} K$-edge of a crystalline stishovite sample (black curve) as reference. Fig. 4(b) depicts the corresponding $\mathrm{Si} L_{2,3}$-edge data.

The collection time was 60 to $90 \mathrm{~s}$ per energy loss point for the Si $L_{2,3}$-edge and $60 \mathrm{~s}$ for the $\mathrm{O} K$-edge. After checking for consistency, we averaged the signals from nine spherically bent analyzer crystals giving an excellent overall data quality. The spectra of the Si $L_{2,3}$-edge at high pressure bear an unprecedented energy resolution of $0.7 \mathrm{eV}$. The statistical accuracy at the $\mathrm{O} K$-edge is high enough to even extract data at different $q$ values.

As the boron-epoxy inset material in the Be gasket contains small amounts of oxygen, the presented $\mathrm{O} K$-edge data have to be analyzed carefully. However, for the extraction of the $\mathrm{O}$ $K$-edge we collected scattering signals from the same detector pixels as for the silicon $L_{2,3}$-edge (shown below), which exhibits little background and, hence, the signal can only originate from the sample and not the sample environment. At 0 and $10 \mathrm{GPa}$, the $\mathrm{O} K$-edge spectra are dominated by a prominent main-edge feature at $538.5 \mathrm{eV}$ energy loss with a small momentum transfer dependent pre-edge shoulder at $536.2 \mathrm{eV}$. After compression to $24 \mathrm{GPa}$, a broad feature around $545 \mathrm{eV}$ 


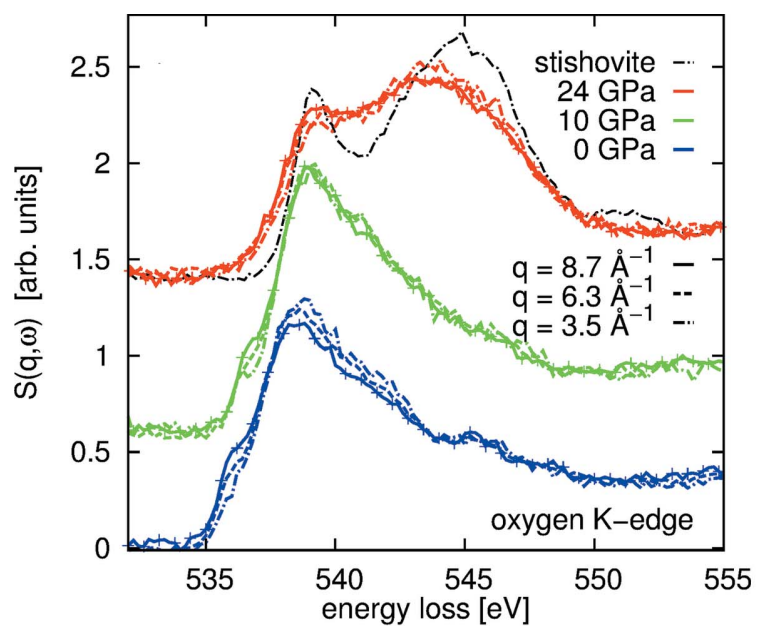

(a)

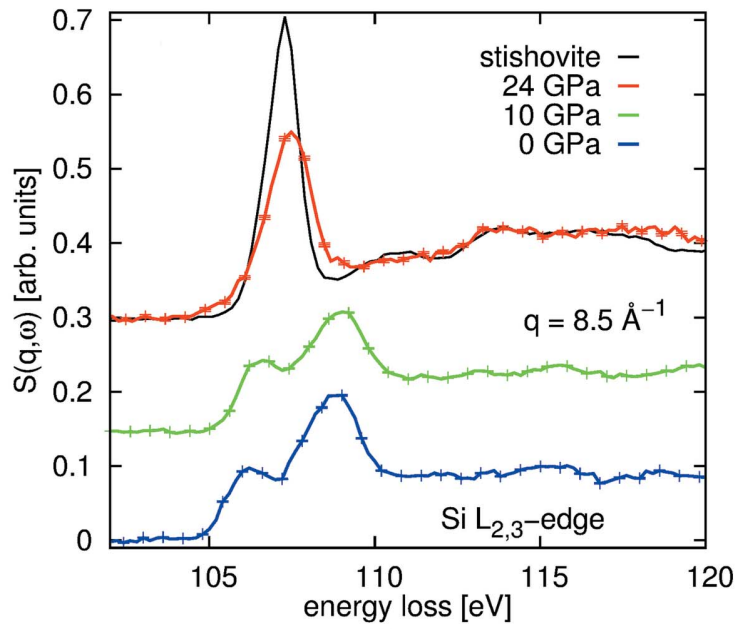

(b)

Figure 4

(a) $\mathrm{O} K$-edges measured at the same pressures as the Si $L_{2,3}$-edge data extracted for momentum transfers of 8.7, 6.3 and $3.5 \AA^{-1}$. (b) Si $L_{2,3}$-edge data from quartz after compression to 0,10 and $24 \mathrm{GPa}$, measured at a momentum transfer of $8.5 \AA^{-1}$. The data were extracted after the described refinement of the ROI.

emerges that was assigned to a transition to sixfold coordinated $\mathrm{Si}$ as reported by Lin et al. (2007). However, as shown for $\mathrm{SiO}_{2}$ glass by $\mathrm{Wu}$ et al. (2012), this feature may only represent an increase of the coordination number in the first coordination shell of $\mathrm{Si}$, but not necessarily a complete transition to a sixfold coordination. Indeed, in comparison with the spectrum of the sixfold coordinated reference stishovite sample, the spectrum of the pressurized quartz sample shows less well defined peaks at 538.5 and 545.0 eV energy loss. From our data, we can assume that quartz undergoes a change to higher coordination of silicon, similar to the sixfold structure of stishovite. We cannot rule out that this structure is only a fivefold or a mixture of five- and sixfold coordination and a deeper understanding would require more data and modeling to elucidate this point. However, this change to higher coordination takes place at the pressure-induced amorphization transition and is most certainly linked to the collapse of the fourfold coordination of quartz as reported by Hemley et al. (1988).

\subsection{Si $L_{2,3}$-edge}

Naturally, one expects the Si $L_{2,3}$-edge to be a clear probe of the coordination of Si. Unfortunately, previous XRS spectra of the Si $L_{2,3}$-edge for pressures up to $75 \mathrm{GPa}$ have shown no significant spectral variation (Fukui et al., 2008), possibly due to the low energy resolution of $2.0 \mathrm{eV}$. In contrast, the Si $L_{2,3^{-}}$ edge spectra shown in Fig. 4(b) reveal a clear change from a two peak structure at 0 and $10 \mathrm{GPa}$ typical for the spectrum of fourfold coordinated $\mathrm{Si}$ (e.g. in $\alpha$-quartz) toward a one peak structure at $24 \mathrm{GPa}$ typical for the spectrum of higher coordinated $\mathrm{Si}$ (e.g. stishovite). These clear changes are visible due to the improved overall energy resolution and statistical accuracy, fit into the context of other experimental data on the glassy counterpart, e.g. using XRD (Sato \& Funamori, 2008, 2010), and hold tremendous promise to finally solve the conundrum of the high-pressure structural phase transition in quartz, silica glasses and other Si bearing compounds such as $\mathrm{MgSiO}_{3}$ glass. Similar to the data of the O $K$-edge, the Si $L_{2,3^{-}}$ edge at the highest probed pressure also shows apparent differences to the $L_{2,3}$-edge spectrum of the stishovite reference sample as a sign of an incomplete transition.

\section{Summary and outlook}

In this study, we presented a scheme to conduct XRS spectroscopy experiments from diamond anvil cells using the direct tomography imaging technique. This allowed us to carefully separate the desired scattering signal of the small sample from undesired scattering of the complicated sample environment. This approach is useful both as an easy tool to align the small sample contained inside the diamond anvil cell in the incident X-ray beam and to analyze the recorded data in a spatially resolved manner. To fully separate scattering intensity originating from sample and sample environment even in delicate cases where core edges of sample and sample environment fully or partly overlap, we used the inherent imaging capabilities of spherically bent analyzer crystals in combination with two-dimensional detectors and a simple data mining approach based on a matrix factorization scheme. We demonstrated the feasibility of the method by showing unprecedented high quality data of the $\mathrm{Si} L_{2,3^{-}}$and $\mathrm{O} K$-edges under pressures of up to $24 \mathrm{GPa}$, with an energy resolution of $0.7 \mathrm{eV}$.

The presented technique and data hold tremendous promise for future in situ XRS experiments on disordered materials under extreme conditions such as high pressure and high temperature.

\section{Acknowledgements}

We kindly acknowledge the ESRF for providing synchrotron radiation and technical support. We thank Michael Krisch for supporting this work and Christian Henriquet for expert technical help and advice. Natalia Dubrovinskaia is kindly acknowledged for making available the stishovite reference sample. 


\section{References}

Beck, P., Gillet, Ph., El Goresy, A. \& Mostefaoui, S. (2005). Nature (London), 435, 1071-1074.

Benmore, C. J., Soignard, E., Amin, S. A., Guthrie, M., Shastri, S. D., Lee, P. L. \& Yarger, J. L. (2010). Phys. Rev. B, 81, 054105.

Boehler, R. \& De Hantsetters, K. (2004). High Pressure Res. 24, 391396.

Chow, P., Xiao, Y. M., Rod, E., Bai, L. G., Shen, G. Y., Sinogeikin, S., Gao, N., Ding, Y. \& Mao, H.-K. (2015). Rev. Sci. Instrum. 86, 072203.

Daniel, I., Gillet, P., McMillan, P. F., Wolf, G. \& Verhelst, M. A. (1997). J. Geophys. Res. 102, 10313-10325.

Fukui, H., Kanzaki, M., Hiraoka, N. \& Cai, Y. Q. (2008). Phys. Rev. B, 78, 012203.

Hemley, R. J., Jephcoat, A. P., Mao, H. K., Ming, L. C. \& Manghnani, M. H. (1988). Nature (London), 334, 52-54.

Hemley, R. J., Mao, H. K., Bell, P. M. \& Mysen, B. O. (1986). Phys. Rev. Lett. 57, 747-750.

Henderson, G. S., de Groot, F. M. F. \& Moulton, B. J. A. (2014). Rev. Mineral. Geochem. 78, 75-138.

Hiraoka, N., Fukui, H. \& Okuchi, T. (2016). High Pressure Res. 36, 250-261.

Hiraoka, N., Fukui, H., Tanida, H., Toyokawa, H., Cai, Y. Q. \& Tsuei, K. D. (2013). J. Synchrotron Rad. 20, 266-271.

Huotari, S., Pylkkänen, T., Verbeni, R., Monaco, G. \& Hämäläinen, K. (2011). Nat. Mater. 10, 489-493.

Inkinen, J., Niskanen, J., Talka, T., Sahle, Ch. J., Müller, H., Khriachtchev, L., Hashemi, J., Akbari, A., Hakala, M. \& Huotari, S. (2015). Sci. Rep. 5, 15851.

Kubo, T., Kimura, M., Kato, T., Nishi, M., Tominaga, A., Kikegawa, T. \& Funakoshi, K. (2010). Nat. Geosci. 3, 41-45.

Lin, J.-F., Fukui, H., Prendergast, D., Okuchi, T., Cai, Y. Q., Hiraoka, N., Yoo, C.-S., Trave, A., Eng, P., Hu, M. Y. \& Chow, P. (2007). Phys. Rev. B, 75, 012201.

Loa, I., Syassen, K., Monaco, G., Vankó, G., Krisch, M. \& Hanfland, M. (2011). Phys. Rev. Lett. 107, 086402.

Mao, H., Kao, C. \& Hemley, R. J. (2001). J. Phys. Condens. Matter, 13, 7847-7858.

Mao, H. K., Xu, J.-A. \& Bell, P. M. (1986). J. Geophys. Res. 91, 4673.

Mao, W. L., Mao, H.-K., Meng, Y., Eng, P. J., Hu, M. Y., Chow, P., Cai, Y. Q., Shu, J. \& Hemley, R. J. (2006). Science, 314, 636-638.

Meade, C. \& Jeanloz, R. (1988). Science, 241, 1072-1074.

Merkel, S. \& Yagi, T. (2005). Rev. Sci. Instrum. 76, 046109.

Mizuno, Y. \& Ohmura, Y. (1967). J. Phys. Soc. Jpn, 22, 445-449.

Murakami, M. \& Bass, J. D. (2010). Phys. Rev. Lett. 104, 025504.

Murakami, M. \& Bass, J. D. (2011). Proc. Natl Acad. Sci. USA, 108, 17286-17289.

Niskanen, J., Sahle, C. J., Ruotsalainen, K. O., Müller, H., Kavčič, M., Žitnik, M., Bučar, K., Petric, M., Hakala, M. \& Huotari, S. (2016). Sci. Rep. 6, 21012.

Ohtani, E., Ozawa, S., Miyahara, M., Ito, Y., Mikouchi, T., Kimura, M., Arai, T., Sato, K. \& Hiraga, K. (2011). Proc. Natl Acad. Sci. USA, 108, 463-466.
Pedregosa, F., Varoquaux, G., Gramfort, A., Michel, V., Thirion, B., Grisel, O., Blondel, M., Prettenhofer, P., Weiss, R., Dubourg, V., Vanderplas, J., Passos, A., Cournapeau, D., Brucher, M., Perrot, M. \& Duchesnay, E. (2011). J. Mach. Learn. Res. 12, 2825-2830.

Petitgirard, S., Malfait, W. J., Sinmyo, R., Kupenko, I., Hennet, L., Harries, D., Dane, T., Burghammer, M. \& Rubie, D. C. (2015). Proc. Natl Acad. Sci. USA, 112, 14186-14190.

Petitgirard, S., Spiekermann, G., Weis, C., Sahle, C., Sternemann, C. \& Wilke, M. (2017). J. Synchrotron Rad. 24, 276-282.

Ponchut, C., Rigal, J. M., Clément, J., Papillon, E., Homs, A. \& Petitdemange, S. (2011). J. Instrum. 6, C01069.

Pylkkänen, T., Giordano, V. M., Chervin, J. C., Sakko, A., Hakala, M., Soininen, J. A., Hämäläinen, K., Monaco, G. \& Huotari, S. (2010). J. Phys. Chem. B, 114, 3804-3808.

Rosa, A. D., Merkulova, M., Garbarino, G., Svitlyk, V., Jacobs, J., Sahle, Ch. J., Mathon, O., Munoz, M. \& Merkel, S. (2016). High Press. Res. 36, 564-574.

Rueff, J.-P. \& Shukla, A. (2010). Rev. Mod. Phys. 82, 847-896.

Sahle, Ch. J., Mirone, A., Niskanen, J., Inkinen, J., Krisch, M. \& Huotari, S. (2015). J. Synchrotron Rad. 22, 400-409.

Sahle, Ch. J., Schroer, M. A., Juurinen, I. \& Niskanen, J. (2016). Phys. Chem. Chem. Phys. 18, 16518-16526.

Sahle, Ch. J., Sternemann, C., Schmidt, C., Lehtola, S., Jahn, S., Simonelli, L., Huotari, S., Hakala, M., Pylkkanen, T., Nyrow, A., Mende, K., Tolan, M., Hamalainen, K. \& Wilke, M. (2013). Proc. Natl Acad. Sci. USA, 110, 6301-6306.

Sanchez-Valle, C. \& Bass, J. D. (2010). Earth Planet. Sci. Lett. 295, 523-530.

Sanloup, C., Drewitt, J. W. E., Konôpková, Z., Dalladay-Simpson, P., Morton, D. M., Rai, N., van Westrenen, W. \& Morgenroth, W. (2013). Nature (London), 503, 104-107.

Sato, T. \& Funamori, N. (2008). Phys. Rev. Lett. 101, 255502.

Sato, T. \& Funamori, N. (2010). Phys. Rev. B, 82, 184102.

Sato, T., Funamori, N. \& Yagi, T. (2013). J. Appl. Phys. 114, 103509.

Schülke, W. (2007). Electron Dynamics by Inelastic X-ray Scattering. Oxford University Press.

Shieh, S. R., Jarrige, I., Wu, M., Hiraoka, N., Tse, J. S., Mi, Z., Kaci, L., Jiang, J.-Z. \& Cai, Y. Q. (2013). Proc. Natl Acad. Sci. USA, 110, 18402-18406.

Shim, S.-H. \& Catalli, K. (2009). Earth Planet. Sci. Lett. 283, 174-180.

Sternemann, C., Sahle, C. J., Mende, K., Schmidt, C., Nyrow, A., Simonelli, L., Sala, M. M., Tolan, M. \& Wilke, M. (2013). J. Phys. Conf. Ser. 425, 202011.

Sternemann, H., Sternemann, C., Seidler, G. T., Fister, T. T., Sakko, A. \& Tolan, M. (2008). J. Synchrotron Rad. 15, 162-169.

Sternemann, C. \& Wilke, M. (2016). High Pressure Res. 36, 275-292.

Tse, J. S., Hanfland, M., Flacau, R., Desgreniers, S., Li, Z., Mende, K., Gilmore, K., Nyrow, A., Moretti Sala, M. \& Sternemann, C. (2014). J. Phys. Chem. C, 118, 1161-1166.

Tse, J. S., Shaw, D. M., Klug, D. D., Patchkovskii, S., Vankó, G., Monaco, G. \& Krisch, M. (2008). Phys. Rev. Lett. 100, 095502.

Tse, J. S., Yang, L., Zhang, S. J., Jin, C. Q., Sahle, Ch. J., Sternemann, C., Nyrow, A., Giordano, V., Jiang, J. Z., Yamanaka, S., Desgreniers, S. \& Tulk, C. A. (2011). Phys. Rev. B, 84, 184105.

Wu, M., Liang, Y., Jiang, J.-Z. \& Tse, J. S. (2012). Sci. Rep. $2,398$. 